\title{
An Enhanced Approach for Preprocessing of Mammogram Images using Inverse Daubechies Wavelet Transform and Non-Linear Diffusion
}

\author{
S. Kowsalya \\ Research Scholar, \\ Department of Computer Science \\ Karpagam Academy of Higher Education, \\ Coimbatore -21
}

\author{
D. Shanmuga Priyaa, $\mathrm{PhD}$ \\ Professor, \\ Department of Information Technology \\ Karpagam Academy of Higher Education, \\ Coimbatore - 21
}

\begin{abstract}
Breast cancer is the most leading cause of death in women nowadays. Screening mammography is currently the best available radiological technique for early detection of breast cancer. The detection of breast cancer is disturbed due to the existence of artifacts which reduce the rate of accuracy. For this reason, the pre-processing of mammogram images is very important in the process of breast cancer analysis because it reduces the number of false positives. This paper discusses about two existing filtering techniques and compares it with the results of a proposed filtering method. It is used to solve the noise removal problems and separate the background region from the breast profile region using an automatic thresholding technique. The results are evaluated on the pre-processing method on a set of images obtained from MIAS database. Thus this preparation phase improves the image quality and accentuates the CAD results more accurately.
\end{abstract}

\section{General Terms}

Data Mining, Image mining, Breast Cancer Detection, Mammograms.

\section{Keywords}

Breast Cancer Detection, Mammogram images, pre-processing, image de-noising, filtering, breast contour detection, pectoral muscle extraction, Inverse Daubechies wavelet transform, nonlinear diffusion.

\section{INTRODUCTION}

To determine the orientation of the mammogram and denoising to enhance the quality of images pre-processing techniques plays a vital role [3]. Before any image-processing algorithm can be applied on mammogram it is essential to perform preprocessing steps which frontier explore for deviations devoid of excessive persuade from background of the mammogram. It is difficult to interpret digital mammograms which are medical images that a preparation phase is required in order to progress the image quality and construct the segmentation consequences further precise. The foremost intention of this method is to advance the excellence of the image to build it prepared to promote processing by eliminating the disparate and surplus parts in the background of the mammogram. Extraction of Breast border region and pectoral muscle suppression is also an element of preprocessing. The high intensity rectangular label, low intensity label, tape artifacts are also considered as types of noise observed in mammogram [4].

\section{RELATED WORK}

Image denoising and enhancement are two important functions to be performed in processing an image. Many algorithms have been formulated from time to time to perform the denoising process. Using multi-wavelet with hard threshold method [1] the image is modeled with random variables of Laplace transform. Hard threshold is applied with different frequency levels. The performance of this method is measured in terms of PSNR (Peak Signal to Noise Ratio). This method is both used for noise suppression and image contrast enhancement.

Another novel algorithm called wavelet-based noise model driven denoising algorithm [2] is proposed where good denoising quality can be acquired and also it preserves edges. This method uses the hybrid characteristics of low pass and high pass filtering techniques. The image is first transferred to wavelet components and thresholding is provided. The minimum levels of co-efficients are considered as noise and so maximum co-efficients above the threshold value are omitted for process. Finally an inverse wavelet transform is applied that acquires the resultant image with reduced noise.

In the process of contrast enhancement a modified histogram method [3] is proposed. Initially the low contrast image is enhanced using a modified algorithm of Histogram and it is normalized. For image sharpening a method called homomorphic filtering is used. To measure the performance of image quality Absolute Mean Brightness Error (AMBE) and Entropy is used.

Common denoising methods are Anisotrophic diffusion and Median Filtering [4]. Other preprocessing techniques like power-low transformation and masking provide high enhancement capabilities. Another method called adaptive Weiner Filter can be used in mammogram images to preserve edges and high frequency parts.

Donoho proposed another wavelet method called wavelet shrinkage [5] wherein the input image is decomposed into a 2D version of signal. Using this method the noise gets spread in a uniform manner and it is reduced to less number of co-efficients. Finally inverse wavelet transform is done to denoise the image. Denoising method using Independent Component Analysis [5] is another proposed method that reduces Gaussian noise by providing minimum threshold components of the sparse distribution.

Using Discrete Wavelet Transform method [6] of denoising, the image is decomposed in the form of discrete wavelets and after reconstruction it forms inverse discrete wavelet transform which holds the denoised image. 
In Decision Based Median Filtering [7] method, the first step is to detect the status of pixel whether it is a corrupted one or not. If pixel value is between minimum and maximum value of window to be processed, it is left unchanged, else median value of window is calculated and replaced for the corrupted pixel.

Another new method was proposed by A.Anilet et.al. [8]. They used Wiener filter that estimates cross-correlation and covariance matrices of noisy signals. This resultant image is applied with curvelet transform that retains significant information and removes noise from the image. While performing denoising, the process finds four different types of noise that appears in these mammogram images. They are high intensity label, low intensity label, scanning artifact and tape artifact. The Figure 1 shows the existence of the above said four noises clearly.

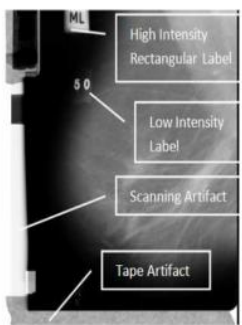

Figure 1 Mammogram image with noise

\section{PROPOSED WORK}

Generally, pre-processing step is composed of two stages: breast region extraction and pectoral muscle extraction. The proposed technique is used to establish an improved preprocessing process in which the images with salt and pepper noise are filtered using fuzzy adaptive filtering.

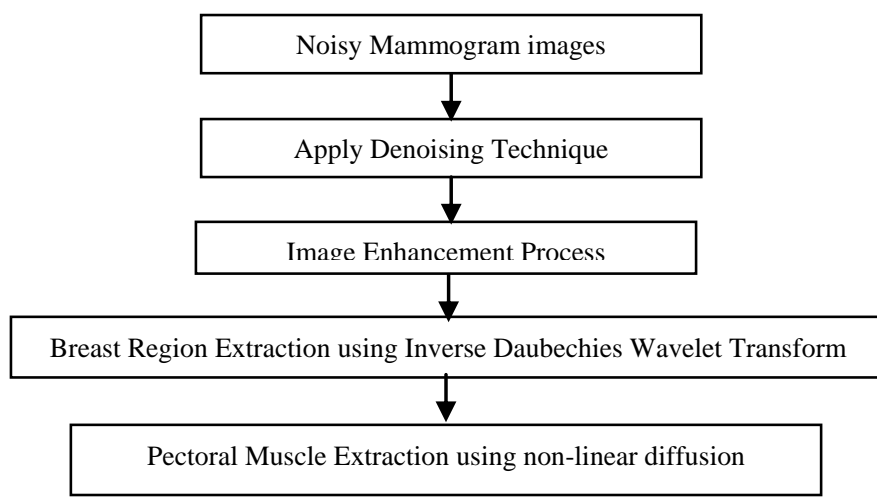

Figure 2 Stages in preprocessing

Figure 2 depicts that the first stage in preprocessing is to apply denoising technique to remove the noise, second stage is the extraction of Breast region using Inverse Daubechies Wavelet Transform which eliminates the background from the breast image and in the third stage elimination of the pectoral muscle from breast region is performed using non-linear diffusion.

The objective of this work is to remove all of the noises from the mammogram images. The acquired results were nearly a faultless rate of removal. Figure 3 shows samples of images in which the first image shows the breast with the presence of noise and the adjacent image appears with the noise removed.
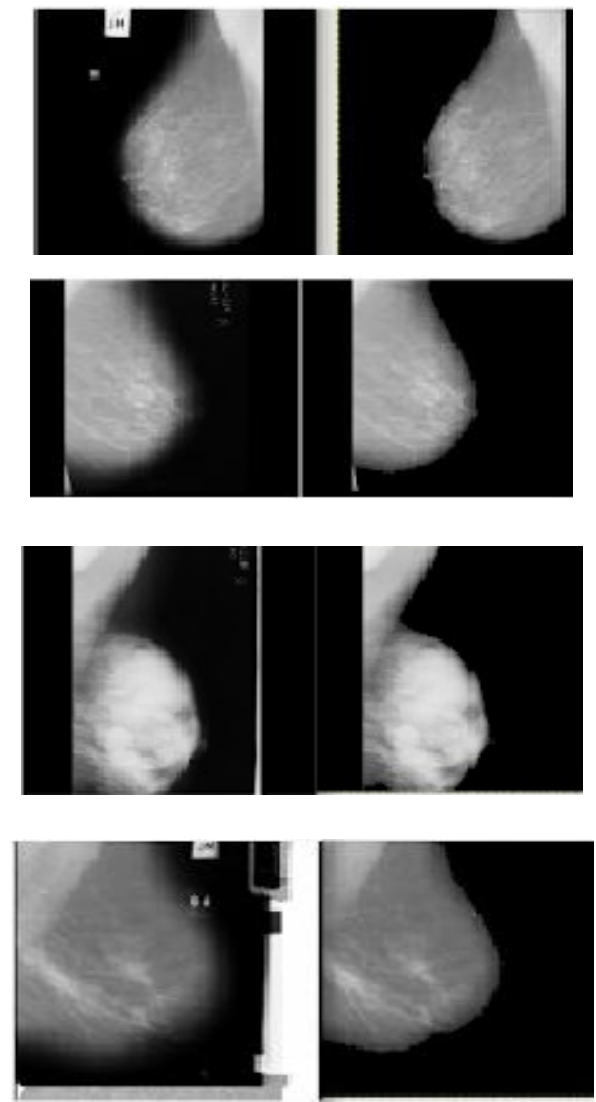

Figure 3 Mammogram Images with and without noise

\subsection{Image Denoising}

Denoising is the process of removing noise from the images before processing them. Many techniques have been followed from time to time. Out of these methods Mean Filtering and Adaptive Filtering techniques are taken into consideration and a new denoising technique called fuzzy adaptive filtering is proposed that shows good performance than the other two filtering technique in the context of PSNR and MSE values.

\subsubsection{Mean Filtering Technique}

This technique finds mean value of the neighbourhood pixels and replaces the existing pixel value from the resultant one. It can nearby condense the difference and is flexible to implement [9]. So this filter is optimal for additive Gaussian noise with respect to mean square error due to its nature of smoothing and blurring the image. If the noise is not of Gaussian type, the simple median filter is not so effective.

\subsubsection{Adaptive Filtering Technique}

The Wiener filter belongs to the type of linear filter when applied adaptively to the images; it is termed as adaptive filtering. During its process it tailors itself to the local image variance. Depending on the variance it performs the smoothing effect low or high [10]. This technique often produces enhanced consequences than linear filtering. While comparing the linear filter, adaptive filter is more selective which preserves edges and other high-frequency parts of mammogram image. This method handles all preliminary computations and implements the filter for an input image. The main pitfall is it require more computation time than linear filtering.

\subsubsection{Fuzzy Adaptive Filtering}

Consider a grayscale image $\mathrm{x}$ termed as an $\mathrm{M} \times \mathrm{N}$ matrix then the intensity of the pixel at the $i^{\text {th }}$ row and the $j^{\text {th }}$ column is represented by $x(i, j)$. The intensity is stored in an 8 -bit integer, 
giving 256 possible gray levels in the interval [0-255]. Maximum and minimum intensity values are taken and this appears in the digital image with equal probabilities. The noise can be either positive or negative [11]. White points are treated as positive impulse and their intensity value is identified as 255 . Black points with negative impulse have intensity value of 0 . Image obtained from first subunit still contains a quantity of noise. Fuzzy member functions are applied to these pixels to denoise the image. If $\mu[\operatorname{IM}(i, j)] \in[0,1]$ is the membership function of IM (i, j) that indicates how much a pixel looks like salt and pepper noise.

Following fuzzy rules can be given:-

[Rule 1] If IM ( $i, j)$ is large, then $\mu$ [IM (i, j)] is large

[Rule 2] If IM ( $i, j)$ is small, then $\mu$ [IM $(i, j)]$ is small

Images for performing denoising process are taken from MIAS database. The mammograms were digitized at 200 micron pixel edge Original images from the database were identified with salt and pepper noise. Experiments were performed with noise of standard deviation of 0.3 times the standard deviation of the original image.

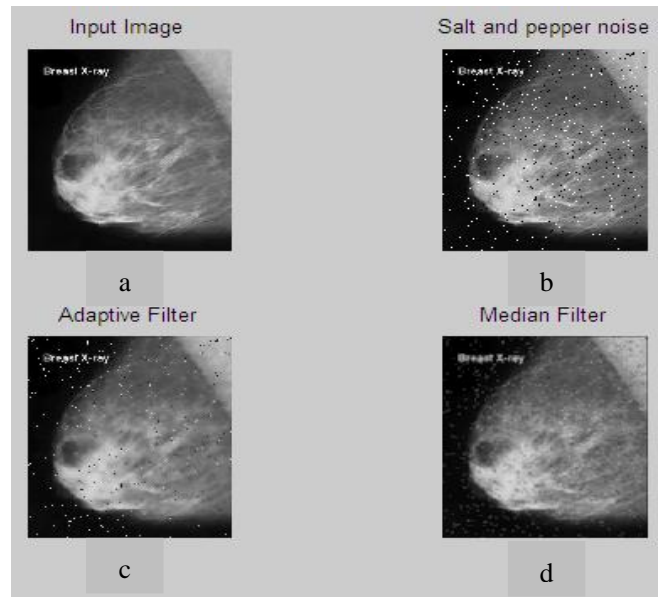

Figure 4 a) Input Image b) Image with Salt and Pepper noise c) Image denoised with Adaptive Filter d) Image denoised with Median Filter

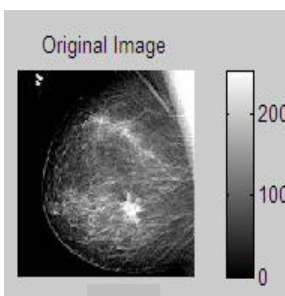

a

$7 \times 7$ Filtered Image

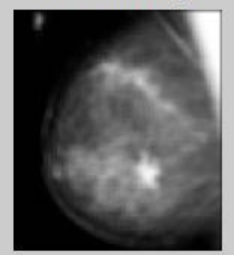

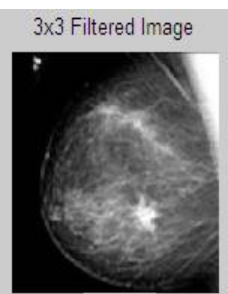

b
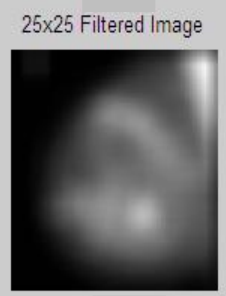

d
Figure 5 a) Original image b) Image with Fuzzy 3x3 Filter c) Image with Fuzzy 7x7 Filter d) Image with Fuzzy 25x25 Filter

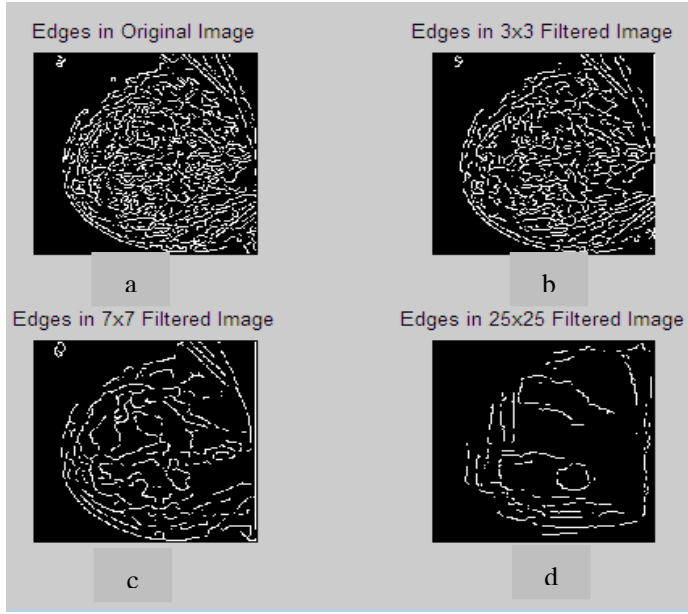

Figure 6 a) Edges in Original image b) Edges in 3x3 Filter c) Edges in 7x7 Filter d) Edges in 25x25 Filter

The Figure 4 shows the image with salt and pepper noise and those removed using Median Filter, Adaptive Filter and Fuzzy Adaptive Filter Figure 5 shows the original image and the denoised image using Fuzzy Filter of matrix size $3 \times 3,7 \times 7$ and $25 \times 25$. Figure 6 shows the original image and edge detected after denoising using Fuzzy Filter of matrix size 3x3, 7x7 and $25 \times 25$.

\subsection{Breast Region Extraction}

\section{Algorithm for extracting the breast contour}

The block diagram for extracting breast contour is shown in Figure 7. A short description of each block is given.

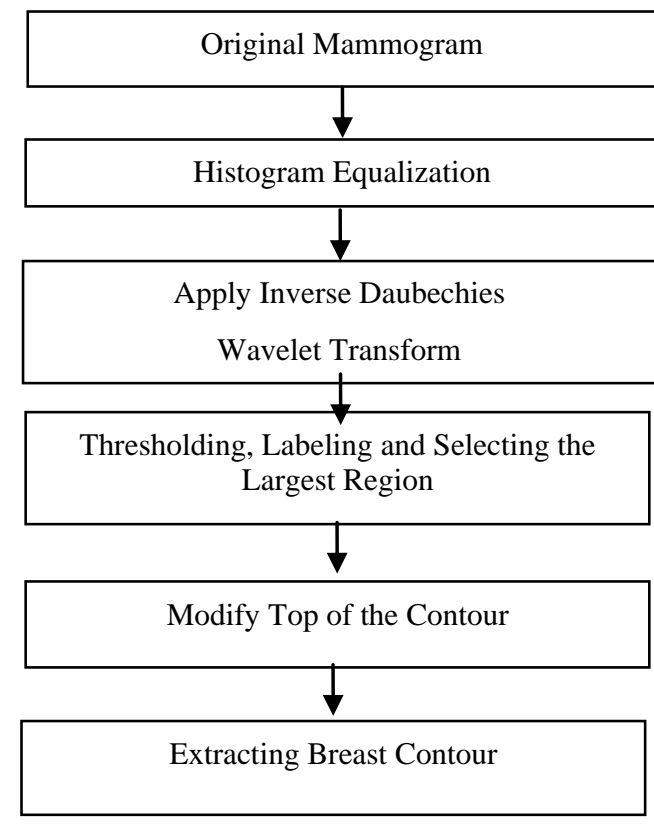

Figure 7 Block diagram for breast region extraction

\subsubsection{Histogram equalization}

Normalization of images becomes an essential process because the proposed algorithm must perform in the same way for all mammograms. So as the first step histogram equalization is done.

\subsubsection{Inverse Daubechies Wavelet Transform}

The inverse of the Daubechies wavelet transform [12, 13], is computed in the reverse order as follows: 


$$
f=\left(\frac{a_{1}-d_{1}}{\sqrt{2}}, \frac{a_{1}+d_{1}}{\sqrt{2}}, \ldots \ldots, \frac{a_{N / 2}-d_{N / 2}}{\sqrt{2}}, \frac{a_{N / 2}-d_{N / 2}}{\sqrt{2}}\right)
$$

To apply Inverse Daubechies wavelet transform on images, first a single level inverse Daubechies wavelet is applied to each column and then to each row of the resulting image.

\subsubsection{Thresholding and labeling}

From the cumulative histogram, a suitable threshold is achieved. As a result a binary image is obtained. Still small noises exist that are removed by applying morphological operations. Larger noises are removed by labeling algorithm. The breast region is subsequently identified as the largest nonzero component. Figure 8 shows the thresholding results using $3 \times 3$ filter, $7 \times 7$ filter and $25 \times 25$ filter.
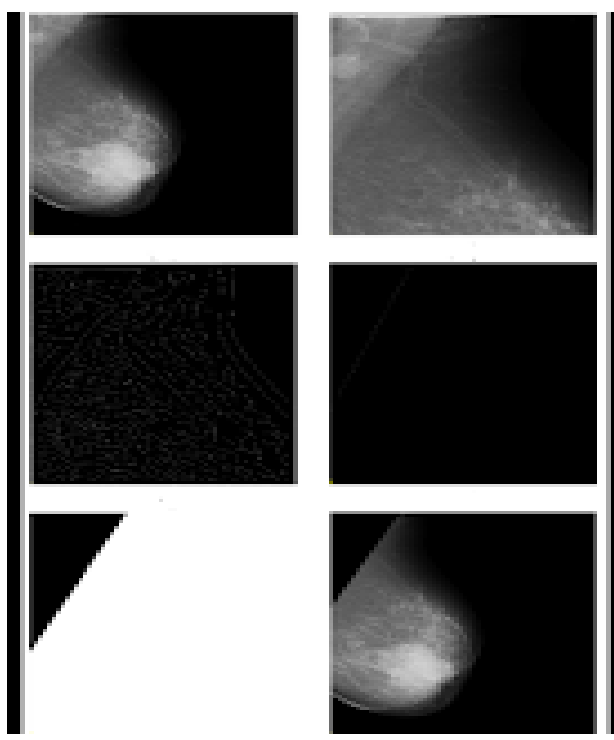

Figure 8 a) Original image b) Thresholding using 3x3 Filter c) thresholding using $7 \times 7$ Filter d) Thresholding using $25 \times 25$ Filter

The algorithm for extraction of breast region is shown below.

\begin{tabular}{|l|}
\hline Algorithm for Breast Region extraction \\
\hline Input: Test image of mammogram \\
Procedure: Stage 1 \\
$\qquad$\begin{tabular}{cc|} 
Steps: \\
$>$ & Select the input image of mammogram \\
$>$ & Perform normalization by applying histogram \\
& equalization on input image \\
$>$ & Transform the image into fourier transform \\
$>$ & Apply Inverse Daubechies Wavelet Transform \\
$>$ & Compute Threshold on the grayscale image \\
& All the pixels with grey level value less \\
than the threshold are marked as
\end{tabular} \\
$>$ background and the rest as breast \\
Output: Extract Breast Contour part alone \\
Extracting Breast Contour
\end{tabular}

\subsection{Pectoral muscle extraction}

In this phase the removal of pectoral muscle from the mammogram images was performed which was an added complex task since of diverse dimensions, shapes and pixel intensities from the muscles in the mammogram images. Hough transform is used to determine the geometrical shapes in mammogram images. In this case it is essential to perceive the straight line that divide the muscle from the breast and implement a mask to remain the image without it. After this the following process are also done

- Smoothing

- Finding the orientation

- Extracting a region of interest that holds the pectoral muscle

- $\quad$ Applying an edge detection filter

The below Figure 9 shows some of the images after extracting the pectoral muscle.
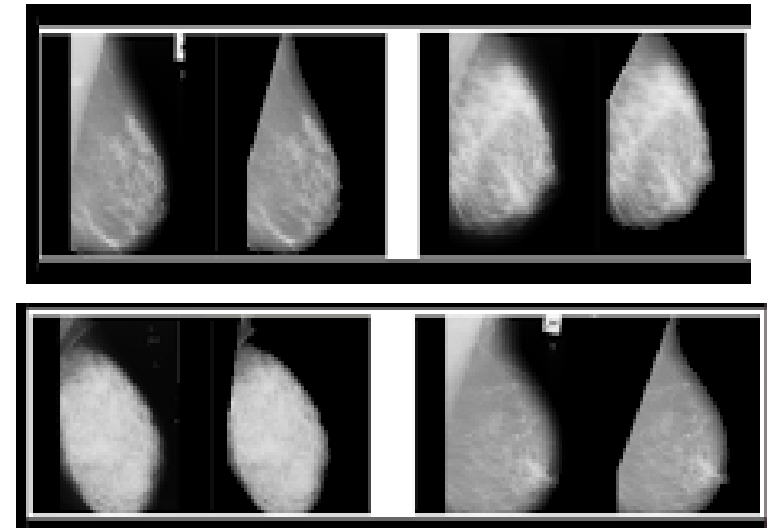

Figure 9 Sample mammogram images with pectoral muscle extracted

In this section the overall method used for pectoral muscle detection is proposed. The flowchart of this method is shown in Figure 10.

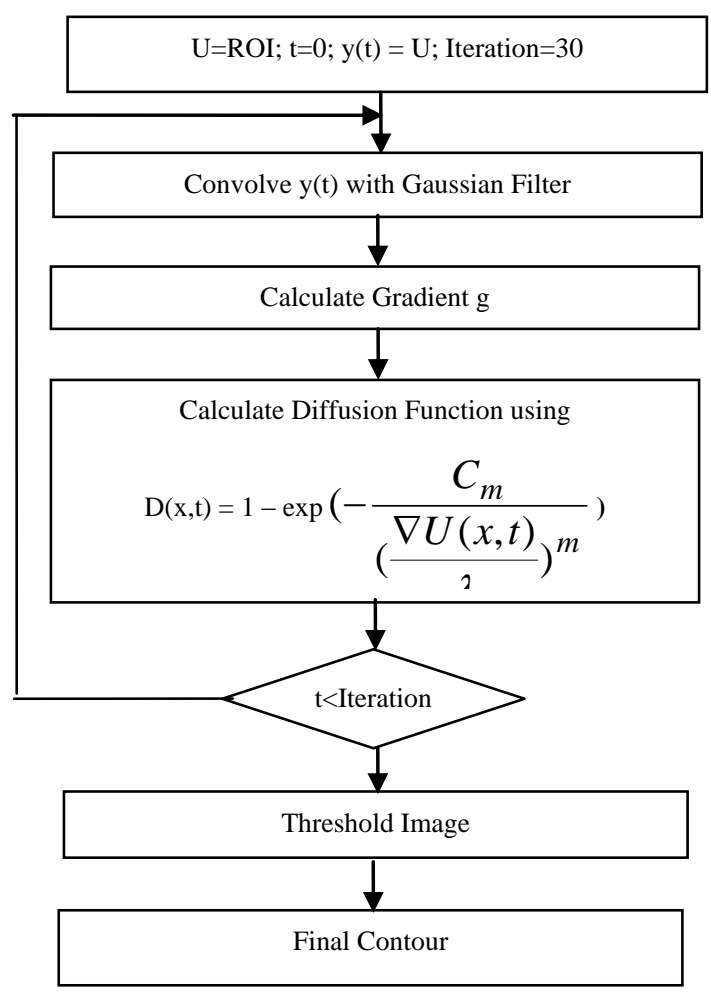

Figure 10 Flow diagram for identification of the pectoral muscle 

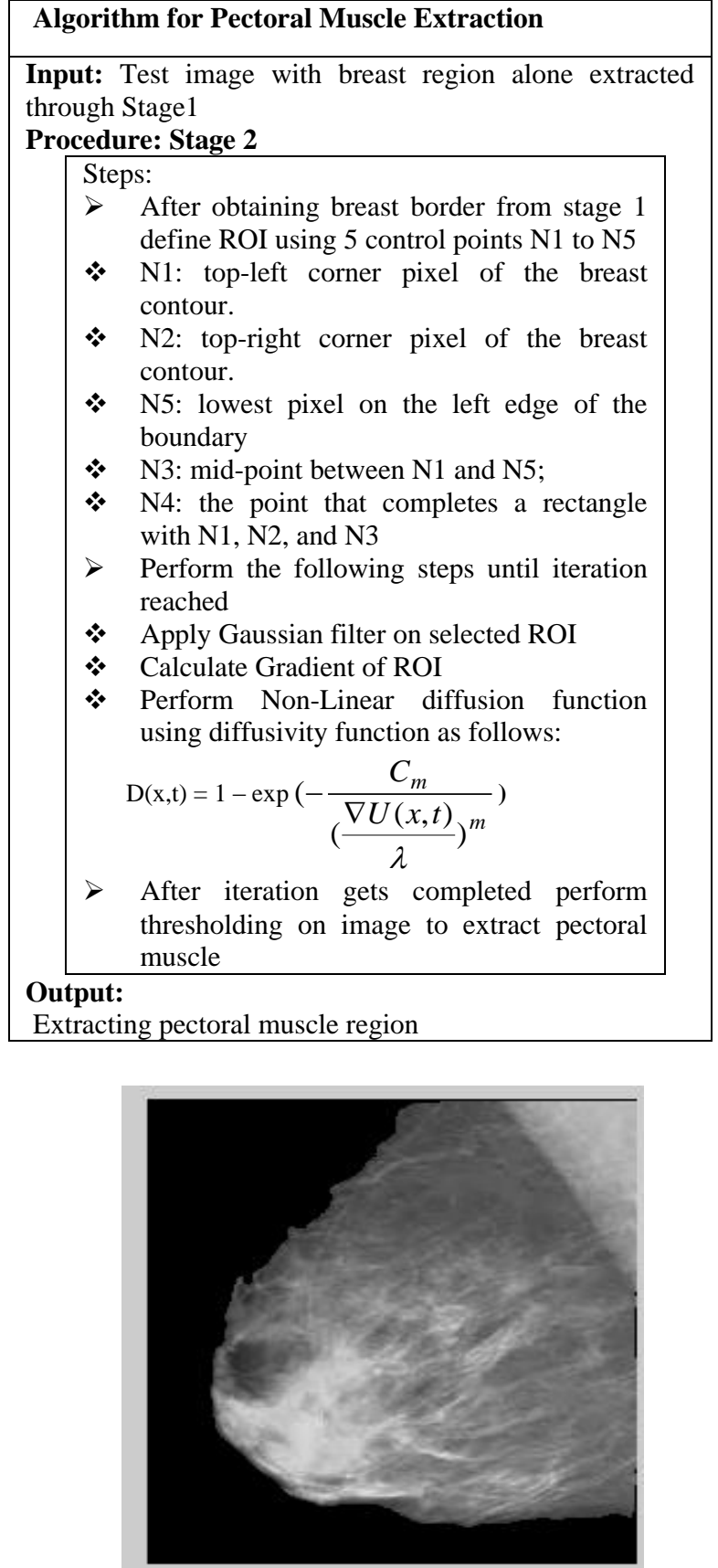

Figure 11 Tag removed image

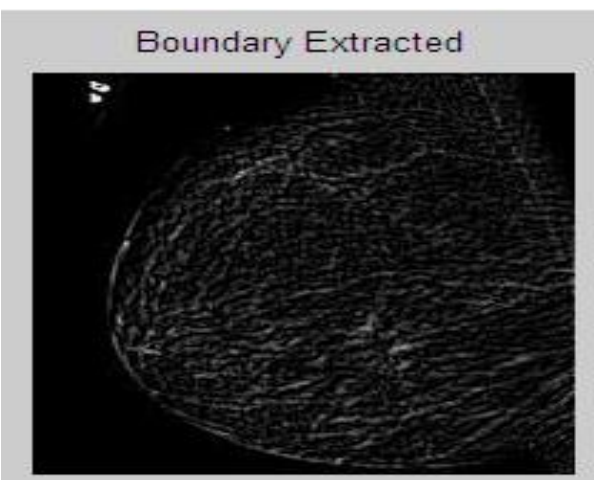

Figure 12 Image after boundary extraction

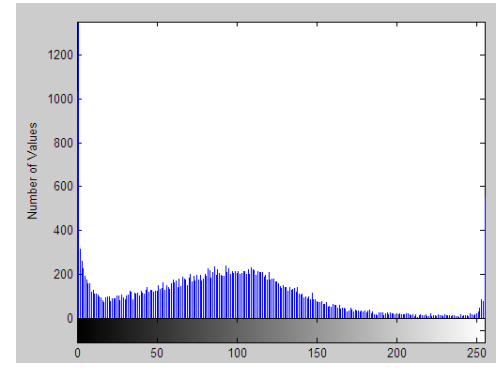

\section{Figure 13 Graphical representation of Pectoral muscle extraction}

Figure 11 and Figure 12 represents the resultant images after performing the pectoral muscle extraction. Figure 13 shows the graphical representation of performance of pectoral muscle removal process after performing denoising.

\section{EXPERIMENTAL RESULTS}

The comparison among the different denoising results was quantitative measured by using the Peak Signal Noise Ratio (PSNR) and Mean Squared Error (MSE) between the denoised image and the original noise free image.

Table.1: Comparison of Denoising Techniques

\begin{tabular}{|c|c|c|c|c|c|c|}
\hline \multirow{2}{*}{ Images } & \multicolumn{2}{|c|}{ Median Filter } & \multicolumn{2}{|c|}{ Adaptive Filter } & \multicolumn{2}{c|}{ Fuzzy Filter } \\
\cline { 2 - 7 } & PSNR & MSE & PSNR & MSE & PSNR & MSE \\
\hline $\begin{array}{c}\text { Image 1 } \\
\text { with } \\
\text { Salt and } \\
\text { Pepper } \\
\text { noise }\end{array}$ & $\begin{array}{c}34.0745 \\
134\end{array}$ & 25.65 & $\begin{array}{c}34.93262 \\
42\end{array}$ & 21.05 & $\begin{array}{c}47.58107 \\
69\end{array}$ & 1.14 \\
\hline $\begin{array}{c}\text { Image 2 } \\
\text { with } \\
\text { Salt and } \\
\text { Pepper } \\
\text { noise }\end{array}$ & $\begin{array}{c}33.6549 \\
43\end{array}$ & 27.09 & $\begin{array}{c}36.89760 \\
3\end{array}$ & 20.06 & 49.89543 & 1.07 \\
\hline $\begin{array}{c}\text { Image 3 } \\
\text { with } \\
\text { Salt and } \\
\text { Pepper } \\
\text { noise }\end{array}$ & $\begin{array}{c}39.0659 \\
987\end{array}$ & 17.49 & 40.09583 & 17.03 & 48.62190 & 1.27 \\
\hline $\begin{array}{c}\text { Image 4 } \\
\text { with } \\
\text { Salt and } \\
\text { Pepper } \\
\text { noise }\end{array}$ & $\begin{array}{c}31.6675 \\
44\end{array}$ & 23.08 & $\begin{array}{c}36.48732 \\
1\end{array}$ & 19.69 & 41.88954 & 1.94 \\
\hline
\end{tabular}

Table.1 shows the comparison of four different mammogram images collected from the MIAS database. Three filtering techniques Median, Adaptive and Fuzzy filter are implemented on salt and pepper noise. The result shows that fuzzy filter performs better than the remaining techniques by holding highest PSNR value and Low MSE Value. The Figure 14 shows the comparison of PSNR values for the three different filters that is Median, Adaptive and Fuzzy. 


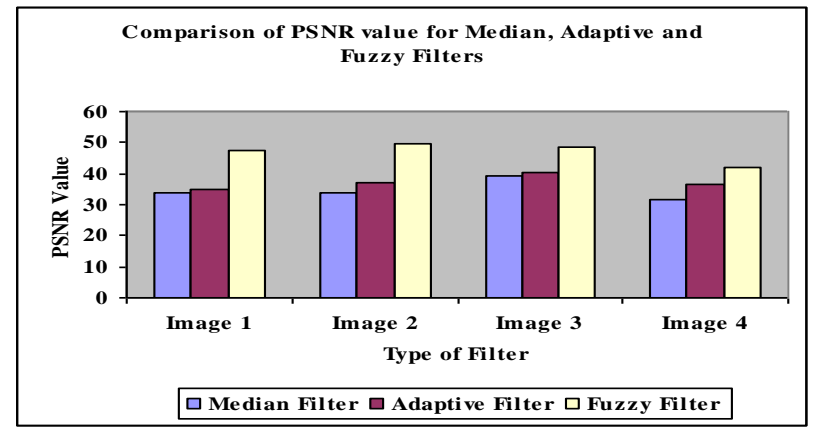

Figure 14 Graphical representation for PSNR Comparison of Median, Adaptive and Fuzzy Filters

The Root Mean Square Error is calculated to evaluate the performance of pectoral muscle extraction before and after denoising the images using fuzzy filters. RMSE is calculated by:

$$
R M S E=\sqrt{\frac{\sum(I(x, y)-\hat{I}(x, y))^{2}}{n-m}}
$$

where $\mathrm{I}(\mathrm{x}, \mathrm{y})$ and $\hat{\mathrm{I}}(\mathrm{x}, \mathrm{y})$ are the pixel values of the original free noise and denoised images respectively, and the size of the image is given by $n * m$.

The Table 2 shows the RMSE value obtained for pectoral muscle detection for four different cases after performing fuzzy filtering.

Table. 2 RMSE value of Pectoral Muscle Detection before and after filtering

\begin{tabular}{|c|c|c|}
\hline \multirow{2}{*}{ Images } & \multicolumn{2}{|c|}{ Root Mean Square Error } \\
\cline { 2 - 3 } & $\begin{array}{c}\text { Before } \\
\text { Filtering }\end{array}$ & $\begin{array}{c}\text { After } \\
\text { Filtering }\end{array}$ \\
\hline $\begin{array}{c}\text { Image 1 with Salt and } \\
\text { Pepper noise }\end{array}$ & 6.520 & 3.773 \\
\hline $\begin{array}{c}\text { Image 2 with Salt and } \\
\text { Pepper noise }\end{array}$ & 8.034 & 4.234 \\
\hline $\begin{array}{c}\text { Image 3 with Salt and } \\
\text { Pepper noise }\end{array}$ & 5.989 & 3.279 \\
\hline $\begin{array}{c}\text { Image 4 with Salt and } \\
\text { Pepper noise }\end{array}$ & 5.087 & 3.095 \\
\hline
\end{tabular}

The below Figure 15 shows the comparison of RMSE values obtained before and after filtering.

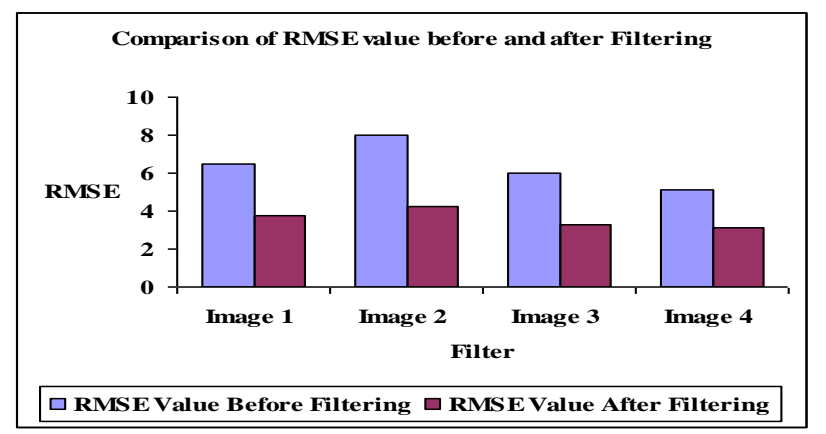

Figure 15 Graphical representation for RMSE Comparison before and after filtering

\section{CONCLUSION}

In breast cancer detection using mammogram image processing plays a key role. To detect the presence of such abnormal symptoms in images several processing has to be undergone to produce a effective result. Since early detection of breast cancer is significant, the preprocessing of mammogram image gains more importance. This proposed work undergoes two stages in the first stage removing of noise using fuzzy filter is carried out to produce a enhanced image. The denoising techniques proposed here are suitable for application to any mammogram image. After denoising in the second stag the breast region is extracted using Inverse Daubechies wavelet transform and the pectoral muscle is detected using non-linear diffusion method. This pectoral muscle alone is considered as ROI for further analysis. This paper have used the Peak Signal Noise Ratio and mean squared error to quantify the success of the three denoising methods namely mean filter, adaptive filter and the proposed fuzzy filter they are applied to Mammographic images. PSNR and MSE are considered to be the standard performance evaluation measures for denoising techniques. The proposed fuzzy filter with non-linear diffusion method breast region is extracted. The results for the four cases are comparable from the PSNR, MSE and visual point of view. The proposed work performs better than existing technique in the presence of salt and pepper noise which commonly appeared in mammogram. The future work will be the automatic segmentation of breast region to determine the presence of masses and calcification in mammogram.

\section{REFERENCES}

[1] Kother Mohideen, Arumuga Perumal, Krishnan, Mohamed Sathik. 2011. Image denoising and enhancement using Multiwavelet with Hard Threshold in Digital Mammographic images, International Arab Journal of eTechnology, Vol. 2, No. 1.

[2] Caroline Arboleda, Zhantian Wang and Macro Stampanoni. 2013. Wavelet based noise-model driven denoising algorithm for differential phase contrast mammography, Optical society of America, Vol. 21, No. 9.

[3] Tarun Kumar Agarwal, Mayank Tiwari, Subir Singh Lamba. 2014. Modified histogram based contrast enhancement using homomorphic filtering for medical images, Advance Computing Conference (IACC), 2014 IEEE International.

[4] Sharma, J, Rai, J.K. ; Tewari, R.P. 2014. Identification of pre-processing technique for enhancement of mammogram images, Medical Imaging, m-Health and Emerging Communication Systems (MedCom), 2014 International Conference.

[5] P.Mayo, F.Rodenas, G. Verdu. Sep 2004. Comparing methods to denoise mammographic images, Proceedings of 25th Annula International Conference of IEEE EMBS.

[6] Taranjot Kaur. 2014. Image denoising Algorithms and DWT: A Review, Int. Journal of Computer science and Information Technologies Vol. 5 No. 5.

[7] Suman Shreshta. 2014. Image denoising using New adaptive Based Median Filter", Int. Journal of Signal and image Processing, Vol. 5, No. 4.

[8] Anilet Bala, chiranjeeb Hati, CH Punith. 2014. Image denoising using Wavelet transform and wiener filter, Int. Journal of advanced Research in Electrical, electronics and Instrumentation Engineering”, Vol. 3 No.1 , Jan 2014. 
[9] Juan Shan, Wen Ju, Yanhui, Guoa, Ling Zhang , H.D. Cheng. 2010. Automated breast cancer detection and classification using ultrasound images: A survey, Pattern Recognition 43299 - 317.

[10] Indra Kanta Maitra, Sanjay Nag, Samir Kumar Bandyopadhyay. 2011. Technique for preprocessing of digital mammogram, computer methods and program and medicine.

[11] Seng Kah Phooi, Man Zhihong, H. R. Wu. 2002. A New Approach in Fuzzy Adaptive Filtering, Fuzzy Logic Studies in Fuzziness and Soft Computing Volume 81, pp 277-287.

[12] R.N. Strick. 1996. Wavelet transforms for detecting microcalcifications in mammograms, IEEE Trans. Med. Imaging Vol. 15 No 2 pp 218-229.

[13] S.N. Yu, K.Y. Li, Y.K. Huang. 2006. Detection of microcalcifications in digital mammograms using wavelet filter and Markov random field model, Comput. Med. Imaging Graphics 30 163-173. 